\title{
Crowd simulation for interactive virtual environments and VR training systems
}

\author{
Branislav Ulicny and Daniel Thalmann \\ Computer Graphics Lab (LIG) \\ Swiss Federal Institute of Technology \\ EPFL, DI-LIG, CH 1015 Lausanne, Switzerland \\ http://ligwww.epfl.ch \\ Branislav.Ulicny@epfl.ch, Daniel.Thalmann@epfl.ch
}

\begin{abstract}
In this paper we present recent results concerning development of the crowd simulation for interactive virtual environments such as virtual reality training system for urban emergency situations. Our system aims to reproduce realistic scenarios involving large number of the virtual human agents with behaviors based on the behaviors of the real persons in such situations. We define architecture of multi-agent system allowing both scripted and autonomous behaviors of the agents as well as interactions among them, with the virtual environment and with the real human participants.
\end{abstract}

Keywords: crowd simulation, multi-agent systems, autonomous agents, VR training systems

\section{Introduction}

Crowds are ubiquitous feature of everyday life. People have long assembled collectively to observe, to celebrate, or to protest at various happenings. The collective assemblages or gatherings called crowds have been the object of scientific inquiry since the end of 19th century [10]. With computers it become possible not only to observe human crowds in the real world, but also to simulate various phenomena from the domain of collective behavior in the virtual environments. Collective behaviors have been studied and modelled with very different purposes. Besides single work concerned with generic crowd simulation [15], most approaches were application specific, focusing on different aspects of the crowd behavior. As a consequence they employ different modelling techniques ranging from those that do not distinguish individuals such as flow and network models, to those that represent each individual as being controlled by rules based on physical laws or behavioral models. Applications include animation production systems used in entertainment industry [4], crowd behavior models used in training of military personnel [20] or policemen [21], crowd motion simulations to support architectural design both for everyday use [3] and for emergency evacuation conditions $[18,19]$, simulations of physical aspects of crowd dynamics [7] and finally sociological and behavioral simulations [12].

The aim of this paper is to introduce recent results concerning development of crowd simulation for interactive virtual environments with application as a training system for urban emergency situations. Goal of the simulation is to reproduce realistic scenarios of such situations evolving in real-time involving large number of virtual human agents. In the next section we describe requirements and constrains for such system and then we define architecture of multi-agent system allowing both scripted and autonomous 
behaviors of the agents as well as their interactions with the virtual environment and immersed users. Next the behavior model is discussed in more details and finally before conclusion we present some early results of the simulation.

\section{Requirements and constrains}

Compared to the simulations of single virtual human, multi-agent systems pose different requirements and constrains for the design of the system in both conceptual and technical aspects. Main conceptual difference is the need for a variety of individual agents' visualizations and behaviors, for example variety of individual trajectories for the group traveling along the same path, variety of the animations for agents having same behavior or different reactions of individuals facing the same situations. Otherwise crowd composed of the same individuals with the same behaviors wouldn't be convincing even if each of such individuals would alone be very realistic. Main technical challenge is increased demand of computational resources which grows in some aspects linearly (for example in agent-environment interactions) but in many quadraticaly (for agent-agent interactions, such as collision avoidance) with the number of simulated agents.

Therefore designing multi-agent simulation is not straightforward task of combining simulations of many single agents, new approaches are required which allow both variety among single agents and in the case of real-time simulations are also computationaly less demanding. Further discussion concerning application specific requirements for crowd modelling can be found in [16].

\section{System design}

Our simulation consists of autonomous virtual human agents ${ }^{1}$ existing in dynamic virtual 3D environment (see figure 1). In order to behave in believable way these agents must act in accordance with their surrounding environment, be able to react to its changes, to the other agents and also to the actions of real humans interacting with the virtual world.

Agents have 3D graphic body representations, which are able to perform certain low-level actions, such as playing of pre-recorded animation sequences (e.g. gestures, changes of postures, etc.) or walking to specified location with different gaits [2]. Highlevel behaviors are then composed of particular combinations of these low-level actions using hierarchical finite state machines. We give more detailed description of the behavior model in the section 4.

Figure 2 gives overview of the system architecture. System is designed with clear separation of the model part (where behavior is computed) from the visualization part (where behavior is displayed), thus allowing use of different virtual humans, objects and environments. In such way it's possible for example to scale up number of the simulated agents by lowering complexity of their 3D representations (e.g. by using levels of details or impostors [1]) without change of the underlying model just by plugging-in different visualization part, or on the other hand simulation can be run without any graphics only with textual output to log file which can later be used to render off-line high quality animations.

\footnotetext{
${ }^{1}$ We distinguish between visualization part of virtual human agent (further refered to as virtual human) and logic part (refered to as agent).
} 


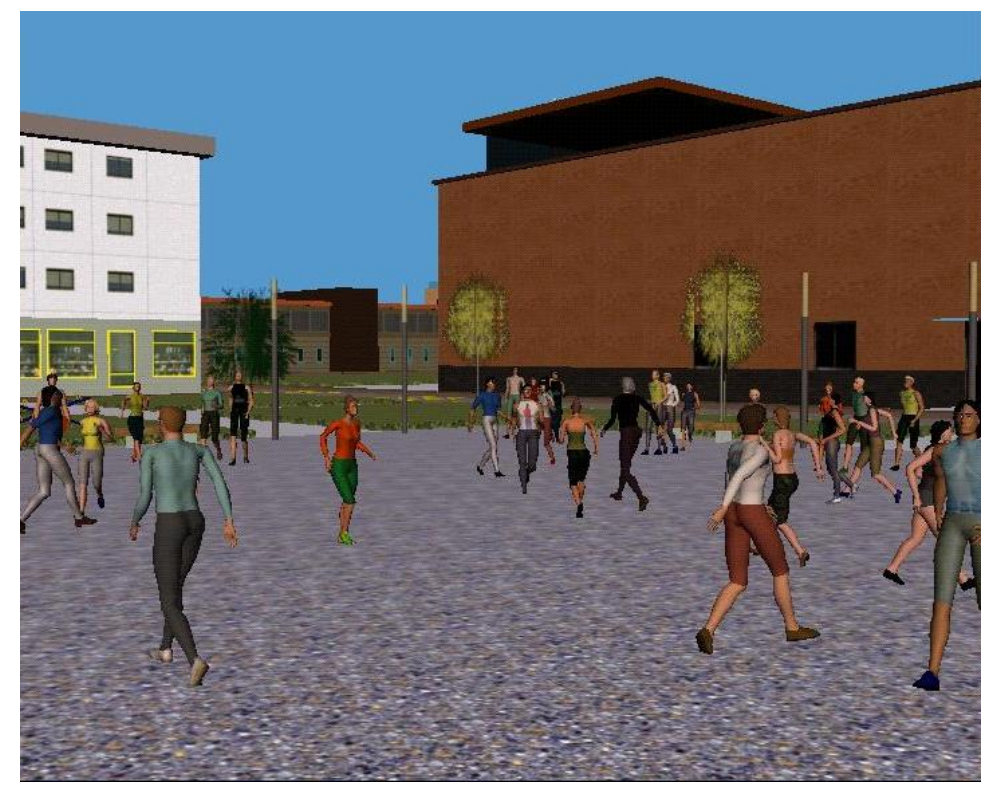

Fig. 1. Virtual environment

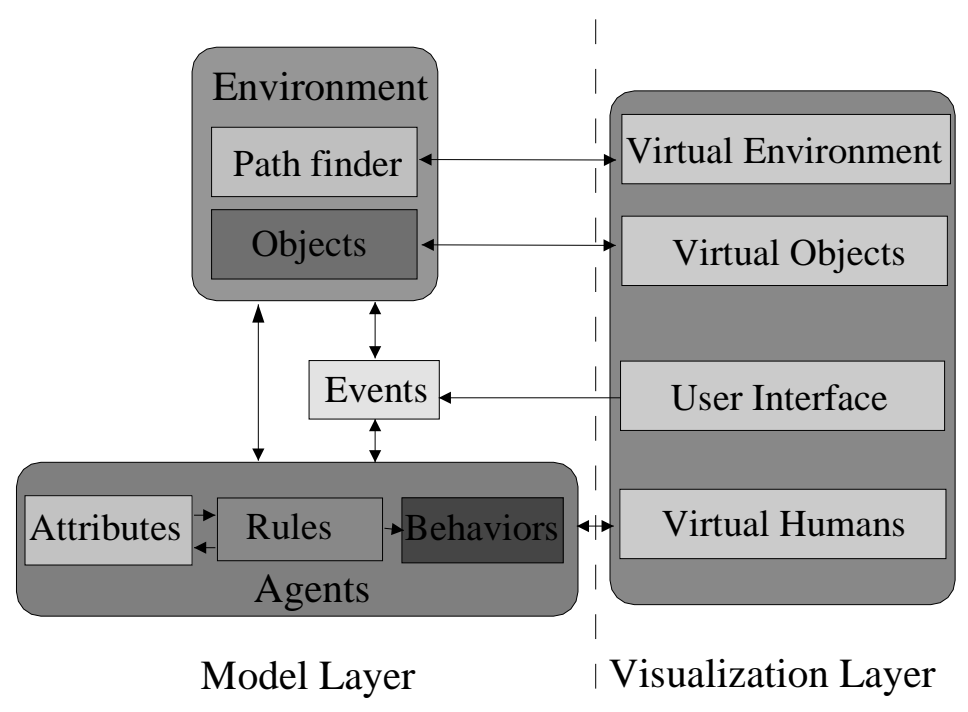

Fig. 2. System architecture 
Model is composed of the set of agents, dynamic objects and static environment ${ }^{2}$. Agents contains set of internal attributes corresponding to various psychological or physiological states (e.g. fear, mobility, level of injuries, etc.), set of higher-level complex behaviors (e.g. wander, flee, etc.) and set of rules determining selection of these behaviors. Events provide way of agents' interaction with their environment, other agents or human participants of the simulation. Each agent is able to receive events from the environmental objects (e.g. agent hit by explosion), other agents (e.g. agent is requesting help) or user interface (e.g. order to stop walking). Combinations of different received events and different levels of agent's attributes can produce both changes of its internal attributes and change of the overt behavior. Further appearance of the agents can be linked to the values of some attributes (e.g. different textures can correspond to different levels injuries). For global path planning in static environment path finder module is used [6]. In case more agents are following the same global path, variety of the individual local trajectories is ensured by specifying path waypoints not by exact location, but by selecting random locations from some epsilon surrounding of the waypoint.

\section{Behavior model}

Our aim is to have behavior model that is simple enough to allow for real-time execution of many agents, yet still sufficiently complex to provide interesting behaviors. Considering requirements mentioned in the section 2 we proposed following model (see figure 3 ) based on the combination of rules [9, 17] and finite state machines (FSM) [5, 13, 14] for controlling agent's behavior using layered approach. First layer deals with the selection of higher-level complex behavior appropriate to agent's situation, second layer implements these behaviors using low-level actions provided by the virtual human [2].

At the higher level, rules select complex behaviors (such as flee) according to agent's state (constituted by attributes) and the state of the virtual environment (conveyed by events). In rules we specify for who (e.g. particular agent, or agents in particular group) and when the rule is applicable (e.g. at defined time, after receiving event or when some attribute reached specified value), and what is the consequence of rule firing (e.g. change of agent's high-level behavior or attribute). Example of such rule is:

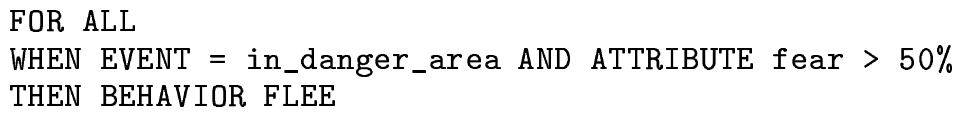

At the lower level, complex behaviors are implemented by hierarchical finite state machines. Each behavior is realized by one FSM which drives selection of the low-level actions for the virtual human (like move to location, play short animation sequence), manages connections with the environment (like path queries, or event sending) and also can call other FSMs to delegate subtasks such as path following ${ }^{3}$.

There are two types of complex behaviors. First we can specify scripted behavior which is more precise, but less autonomous and with less environment coupling by using explicit sequences of low-level actions. Or second we can let agents perform autonomously complex behaviors with the feedback from the environment. Examples

\footnotetext{
${ }^{2}$ We distinguish between static part of the environment like layout of the streets and buildings and dynamic part consisting of objects that can change their position or state during the scenario like fire or gas cloud.

${ }^{3}$ Hence hierarchical FSM.
} 


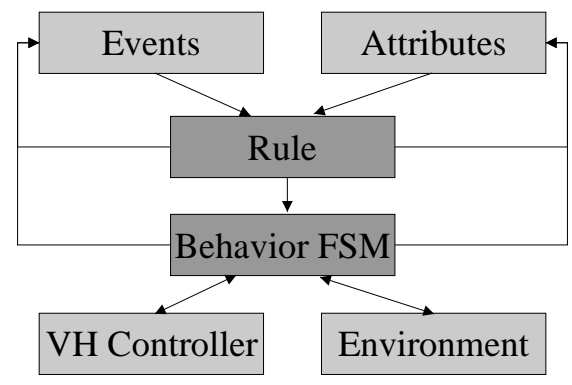

Fig. 3. Behavior model

of such autonomous behaviors are wandering, fleeing, neutralizing the threat, or help requesting and providing.

We can illustrate semantics of the autonomous behaviors on the example of two agents performing coupled behaviors - help requesting and providing. When first agent's health attribute is below certain level, rule triggers help requesting behavior FSM. Agent stops on the place and starts to play animation corresponding to asking for the help (such as waving of the hand) and sends event conveying his request to the other agents. Reception of this event leads to the activation of the rule for the second agent, which then starts performing help providing behavior FSM. He asks environment for the path leading to the first agent, executes path following FSM to perform movement to the injured agent and after arriving he starts playing help giving animation (such as giving first aid). Upon finishing animation first agent is notified and the level of his health attribute is increased. At the end both involved agents quit these behaviors and return to their previous behaviors.

Variety of the reactions to the same situation is achieved by different agents having different values of the attributes (at the beginning through different initializations, later because of their different histories) which consequently leads to different rules triggered. This behavior architecture also addresses variety of the animations issue (see section 2) by separating action selecting part (behavior FSM) from action executing part (virtual human controller). Behavior FSM is ordering controller to do type of the animation (e.g waving of hand) and controler then randomly chooses particular one from the set of such animations, so that even if more agents are executing the same behavior they don't necessarily act exactly the same.

\section{Early results}

To test feasibility of our approach we used early implementation of our system to reproduce simple scenario of urban emergency situation. Scenario is taking place in virtual city environment where people in the park area are confronted with the leak of dangerous gas (see figure 4). It includes simulation of pre-event, emergency event and postevent behaviors where behavioral rules guide transitions between agents' autonomous behaviors in concordance with the changes of the environment. There are three different groups of agents involved - ordinary people, firemen and medics. According to their 

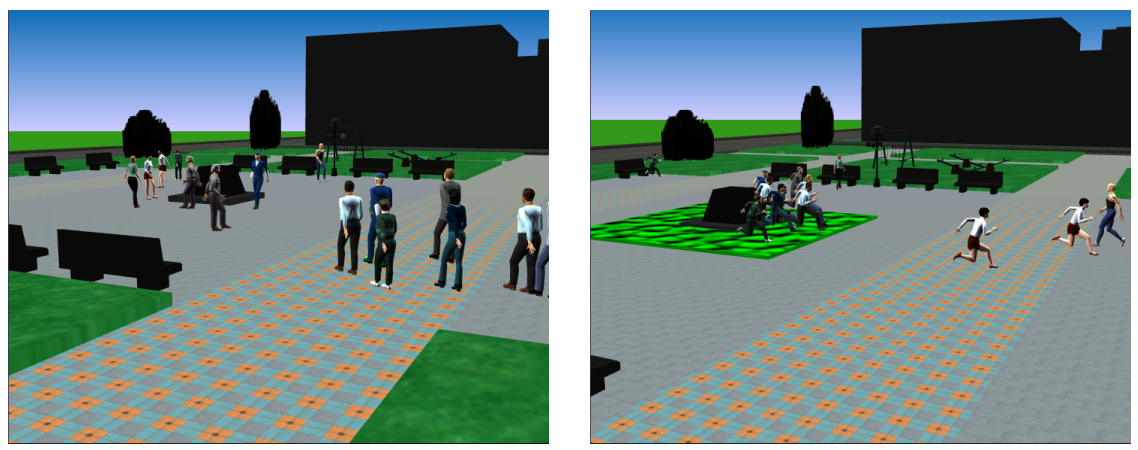

Fig. 4. Virtual world: a) before emergency, b) after gas leak happened

professions agents act differently facing the emergency situation. Ordinary people in proximity of the threat are panicking and trying to flee away, however as gas is affecting them, some are unable to move and are asking for help. In case they stay too long affected by gas their health decreases, eventually leading to death. Medics are localizing such persons and providing help to them. Finally firemen are approaching gas leak and acting towards neutralizing it.

Our system was able to recreate such scenario in real-time allowing user interaction with the virtual world. However major bottleneck proved to be visualization of complex virtual humans. We used three types of the visualization of the agents with different complexities. In the case of simple cube representation we were able to run simulations consisting of up to thousand of agents, for virtual humans with rigid bodies this number dropped to around hundred and finally with the most realistic deformable bodies interactive simulations with about twenty agents were possible ${ }^{4}$.

\section{Conclusions and future work}

This paper presented our work on crowd simulation system for interactive virtual environments. We defined multi-agent architecture allowing virtual human agents to perform autonomous behaviors in the virtual world, where both individual agents and environment are modelled. Agents' behaviors are decided by behavioral rules and executed by hierarchical finite state machines. We used this system to create scenario of urban emergency situation. Possible application of our system could be any virtual environment system requiring real-time execution of complex autonomous behaviors of many agents such as various training systems, computer games or educational applications.

In comparision with the other crowd modelling approaches we focus on more complex behaviors in dynamic environment. In previous works crowds have been considered as already formed units with more or less uniform behavior placed in particular environments corresponding only to limited purpose of simulation e.g. pedestrian just fleeing from burning building [7, 18] or marching crowd during demonstration [20, 21]. In our system crowd is modelled as collection of individuals which reacts to the environment, other agents and real human participants of the simulation and can have very different behaviors both for one agent in different situations and for many agents in the same situation.

\footnotetext{
${ }^{4}$ On SGI Onyx2 workstation.
} 
For the future work we plan to investigate following issues:

- Extension of agent-agent interaction possibilities for enabling of more complex group behaviors.

- Improvement of agent-object interaction and animation for example by using smart objects [8].

- Enhancement of the visual realism of multiple agents performing the same a nimation by using animation system allowing variation over single animation for example by example-based motion synthesis [11].

\section{Acknowledgements}

This work has been supported by the Swiss National Research Foundation and the Federal Office for Education and Science in the framework of the European project CROSSES.

\section{References}

1. Aubel, A., Boulic, R., Thalmann, D., "Real-time Display of Virtual Humans: Level of Details and Impostors", IEEE Trans. Circuits and Systems for Video Technology, Special Issue on 3D Video Technology, 10(2):207-217,2000.

2. Boulic, R., Becheiraz, P., Emering, L., and Thalmann, D., "Integration of Motion Control Techniques for Virtual Human and Avatar Real-Time Animation”, Proc. VRST '97, pp. 111118, ACM Press, 1997.

3. Bouvier, E., Guilloteau, P., "Crowd Simulation in Immersive Space Management", Proc. Eurographics Workshop on Virtual Environments and Scientific Visualization '96, pp. 104110, Springer-Verlag, 1996.

4. Character Studio 3, data sheet, http://www2.discreet.com/docs/characterstudio3_1.pdf, 2001.

5. Cremer, J., Kearney, J., and Papelis, Y., "HCSM: Framework for Behavior and Scenario Control in Virtual Environments", ACM Transactions on Modeling and Computer Simulation, 5(3):242-267, 1995.

6. Farenc, N., Boulic, R., Thalmann, D., "An Informed Environment Dedicated to the Simulation of Virtual Humans in Urban Context”, Proc. Eurographics'99, pp. 309-318, Blackwell, 1999.

7. Helbing, D., Farkas, I., and Vicsek, T., "Simulating dynamical features of escape panic", Nature, 407:487-490, 2000.

8. Kallmann, M., and Thalmann, D., "Modeling Objects for Interaction Tasks", Proc. Eurographics Workshop on Computer Animation and Simulation '98, pp. 73-86 Springer-Verlag, 1998.

9. Kalra, D., Barr, A.H., Modeling with Time and Events in Computer Animation, Proc. Eurographics'92, pp. 45-58, Blackwell, 1992.

10. LeBon, G., Psychologie des Foules, Paris:Alcan, 1895.

11. Lim, I.S., Thalmann, D., "A Vector-Space Representation of Motion Data for ExampleBased Motion Synthesis", Proc. Avatars 2000, Lausanne, Switzerland, 2000.

12. McPhail, C., Powers, W.T., and Tucker, C.W., "Simulating individual and collective actions in temporary gatherings", Social Science Computer Review, 10(1):1-28, Spring, 1992.

13. Moreau, G., Donikian, S., "From Psychological and Real-Time Interaction Requirements to Behavioral Simulation”, Proc. Eurographics Workshop on Computer Animation and Simulation'98, Springer-Verlag, 1998.

14. Motivate product information, Motion Factory, http://www.motion-factory.com. 
15. Musse, S.R., Human Crowd Modelling with Various Levels of Behaviour Control, PhD thesis, EPFL, Lausanne, 2000.

16. Musse, S.R., Thalmann, D., "From One Virtual Actor to Virtual Crowds: Requirements and Constraints", Proc. Autonomous Agents'00, pp. 52-53, ACM Press, 2000.

17. Rosenbloom, P.S., Laird, J.E., Newell, A., The Soar papers: Research on Artificial Intelligence, MIT Press, 1993.

18. Still, G., K., Crowd Dynamics, PhD thesis, Warwick University, 2000.

19. Thompson, P.A., Marchant, E.W., "A Computer-model for the Evacuation of Large Building Population”, Fire Safety Journal, 24(2): 131-148, 1995.

20. Varner, D., et al., "UMSC Small Unit Leader Non-Lethal Trainer", in Proc. ITEC'98, 1998.

21. Williams, J.R., A Simulation Environment to Support Training for Large Scale Command and Control Tasks, PhD thesis, University of Leeds, 1995. 